\title{
Editorial: Emerging Technologies for Ubiquitous and Intelligent Infrastructures
}

\author{
Luca De Nardis $^{1} \cdot$ Marina Petrova $^{2}$
}

Published online: 22 March 2017

(C) Springer Science+Business Media New York 2017

\section{Editorial:}

The increasing degree of intelligence, context awareness and interactivity in everyday objects and appliances is rapidly moving the concept of smart environments from labs and research papers out to the real world: application scenarios like Smart Cities, Smart Mobility, Smart Home are indeed driving the research activities in the field of wireless communications. Although a pervasive deployment of connected devices, capable of bringing to life the Internet of Things vision, is indeed a cornerstone in the embodiment of smart environments, bare connectivity is only one piece of the puzzle: technical advancements and scientific breakthroughs are still required in several areas. Fast, reliable communications in scenarios characterized by extremely high density of devices, thanks to advanced signal processing algorithms for physical layer and robust and flexible protocols for MAC and network layers, is definitely a major research topic relevant to smart environments, as shown by thriving activities on 5 th generation wireless systems. Energy efficiency is a second key aspect that is under heavy scrutiny by the research community, given the strict requirements on lifetime and electromagnetic footprint that will be imposed to devices. Algorithms and protocols supporting the collection of information about the users and their interaction with the environment, e.g. their position, are also expected to play a key role in enabling smart interactions. Finally, security is an open issue that calls for extensive

Luca De Nardis

luca.denardis@uniroma1.it

1 DIET Department, Sapienza University of Rome, Rome, Italy

2 Faculty of Electrical Engineering and Information Technology, RWTH Aachen University, Aachen, Germany

analysis and new proposals at all layers of the protocol stack, given the personal nature of interaction between the user and the environment, and the corresponding sensitivity of the data being collected and exchanged.

The six selected papers forming this special issue addressed aspects related to the topics identified above. The first article, "Joint Atomic Norm based Estimation of Sparse Time Dispersive SIMO Channels with Common Support in Pilot Aided OFDM Systems", proposed a new channel estimation method for OFDM wireless systems equipped with multiple antennas, considering both the case in which multiple antennas are only available at the receiver (SIMO) and the more general MIMO case where multiple antennas are available at both sides. The approach proposed in the paper takes advantage of atomic norm minimization and outperforms existing algorithms in low-to-medium SNR regimes.

The second article, titled "Performance Evaluation of Nonprefiltering vs. Time Reversal prefiltering in distributed and uncoordinated IR-UWB Ad-Hoc networks," focuses on another transmission technique that is expected to play a significant role in future wireless systems, that is Ultra Wide Band (UWB), and considers its combination with the Time Reversal (TR) precoding technique. The paper analyzes the performance of TR-UWB systems in a distributed network of uncoordinated devices, and identifies under which scenarios TRUWB can lead to a performance increase when compared to traditional rake receivers.

The next article, with the title "Analysis of Two-Tier LTE network with Randomized Resource Allocation and Proactive Offloading", moved up the protocol stack by addressing the issue of optimal resource allocation in a heterogeneous network composed of multiple tiers, a scenario that is expected to play a key role in $5 \mathrm{G}$ systems. The authors studied a two-tier network, and proposed both a novel radio resource management scheme 
at the lower tier and a novel offloading scheme to move traffic from the higher tier to the lower one, that combined can provide high data rates to high priority users while guaranteeing fair resource access to low priority users.

Energy efficiency is the focus of the fourth article, titled "Energy-Efficient Context Aware Power Management with Asynchronous Protocol for Body Sensor Network." The paper investigated power efficiency issues in wearable devices, and proposed a novel approach combining the adoption of a low-power wake-up radio to avoid the power consumption due to listening and idle periods of the main radio with a context aware power manager that activates sensors according to an activity recognition routine that determines the activity being carried out by the user, leading to a lifetime increase up to a factor of 4 .

Position information is considered a key input in the definition of context aware systems required for the deployment of smart environments. The fifth article, "Virtual and Oriented WiFi Fingerprinting Indoor Positioning based on Multi-Wall Multi-Floor Propagation Models," focused on positioning of wireless devices based on WiFi fingerprinting, and proposed the adoption of an empiric propagation model to reduce the measurement and collection efforts required for the creation of the fingerprinting database while taking into account the impact of user and device orientation. Experimental results highlight the good accuracy of the model in generating virtual fingerprints.

The last article, titled "Analysis of the Impact of AuthRF and AssRF Attacks on IEEE 802.11e-based Access Point," focused on security aspects by investigating the impact of Denial of Service (DoS) attacks at the MAC layer in IEEE 802.11e compliant systems during the Association and Authentication phases. The paper adopted a detailed analytical model for an $802.11 \mathrm{e}$ considered as a queueing system, and investigated the impact of DoS attacks as a function of offered traffic rates, highlighting the strong dependency of the impact of an attack on the characteristics of the offered traffic. The paper reviewed as well the most promising techniques to mitigate the impact of DoS attacks.

Acknowledgement The guest editors are thankful to the reviewers for their effort in reviewing the manuscripts. We also thank the editorial staff and the Editor-in-Chief for their supportive guidance during the entire process.

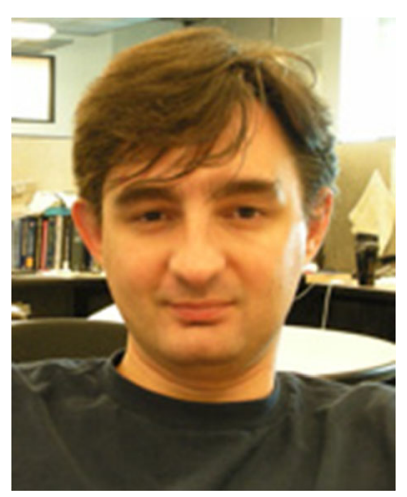

Luca De Nardis received both his Laurea degree and his Ph.D. from Sapienza University of Rome in 2001 and 2005, respectively. Since December 2008 he is an Assistant Professor at the DIET Department of Sapienza University of Rome. In 2006 he was a visiting scholar at the EECS Department at the University of California at Berkeley. He was a postdoctoral fellow at the same institution until April 2007. He authored or coauthored over 100 publications in international peer-reviewed journals and conferences, including IEEE Journal of Selected Areas in Communications, IEEE Transactions on Microwave Theory and Technique and ACM Mobile Networks and Applications. Since 2002, he has been participating in European IST projects focusing on Ultra Wide Band communication systems and cognitive radio, and more recently in COST Actions IC0902 and IC0905 and in the European Network of Excellence ACROPOLIS, all focusing on cognitive radio and networks. His research interests focus on UWB radio technology, Medium Access Control, routing and positioning protocols for wireless networks, and on the design of cognitive wireless networks.He is an IEEE member since 1998.

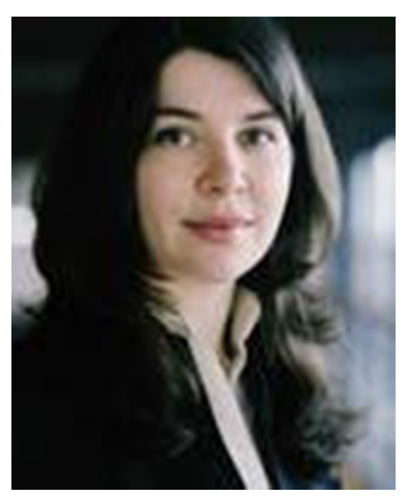

Marina Petrova is an assistant professor at the Faculty of Electrical Engineering and Information Technology at RWTH Aachen University. The professorship is associated with the UMIC research center. She is also a chief research scientist at the Institute for Network Systems. Her research interests are focused on cognitive radios and cognitive wireless networks, adaptive wireless systems technologies and resource optimization problems in wireless networks. Dr. Petrova has actively participated in a number of EU funded international cooperative projects and industry projects in the field of wireless communications and cognitive radios. Moreover, she is also involved in research activities towards prototype implementation of resource management solutions for cognitive radios. Dr. Petrova holds a degree in electrical engineering and telecommunications from University Ss. Cyril and Methodius, Skopje, Macedonia and a Ph.D from RWTH Aachen University, Germany. She has served in technical program committees of numerous IEEE conferences and workshops. She was a TPCco chair of DySPAN 2011. 